\title{
Radio spectral index study of the SNRs OA184 and VR042.05.01
}

\author{
D. Leahy ${ }^{1}$ and W. Tian ${ }^{1,2}$ \\ 1 Department of Physics \& Astronomy, University of Calgary, Calgary, Alberta T2N 1N4, Canada \\ e-mail: wtian@ucalgary.ca \\ 2 National Astronomical Observatories, CAS, Beijing 100012, PR China
}

Received 10 May 2005 / Accepted 2 June 2005

\begin{abstract}
New images of the Supernova Remnants (SNRs) OA184 and VRO42.05.01 are presented at $408 \mathrm{MHz}$ and $1420 \mathrm{MHz}$, from the Canadian Galactic Plane Survey (CGPS) data. The SNRs' flux densities at both $408 \mathrm{MHz}$ and $1420 \mathrm{MHz}$ are found and corrected for flux densities from compact sources within the SNRs. The integrated flux density based spectral indices $\left(\mathrm{S}_{v} \propto v^{-\alpha}\right)$ are $0.25 \pm 0.03$ for OA184 and $0.36 \pm 0.06$ for VRO42.05.01. These agree with the respective $T-T$ plot spectral indexes of $0.23 \pm 0.06$ and $0.36 \pm 0.03$. OA 184 's spectral index is smaller than previously published values. The older flux density values of OA184 from lower resolution data include contributions from a non-SNR ridge emission region and from compact sources within OA184. Subtracting these contributions results in a spectral index of $0.32 \pm 0.06$ ( $38 \mathrm{MHz}$ to $2695 \mathrm{MHz}$ ) or $0.28 \pm 0.06$ (408 MHz to $2695 \mathrm{MHz}$ ). Correction of published flux densities for compact sources for VRO.42.05.01 results in a spectral index of $0.32 \pm 0.05$ for $38 \mathrm{MHz}$ to $2695 \mathrm{MHz}$. We also find spatial variations of spectral index. For OA184 $\alpha$ varies from 0.1 to 0.3 (with errors $\simeq 0.1$ ). For VRO42.05.01, the shell region has $\alpha=0.31$ and the wing region has $\alpha=0.47$ (with errors $\simeq 0.03$ ).
\end{abstract}

Key words. ISM: supervona remnants - ISM: structure

\section{Introduction}

The paper is part of a continuing study of supernova remnants' spectral index variation. The radio spectra of the SNRs OA184 and VRO42.05.01 have not been studied before in detail. However, both SNRs' basic physical features have been studied in previous research.

The distance to OA184 was first suggested to be $8 \pm 2 \mathrm{kpc}$ (Routledge et al. 1986) based on the surface brightnessdiameter $(\Sigma-\mathrm{D})$ relation, then argued to be about $2.6 \mathrm{kpc}$ by Leahy \& Marshall (1988), based on infrared observations. The most reliable estimate is $4.5 \pm 1.5 \mathrm{kpc}$ by Landecker et al. (1989) based on HI measurements. The latter yields a radius for OA184 of $\simeq 50$ pc. OA184 is roughly elliptical in shape in both radio and optical images with similar structure (Routledge et al. 1986) and has no molecular clouds nearby (Huang \& Thaddeus 1986). It is just past the end of the adiabatic phase of evolution (Leahy \& Marshall 1988).

VRO42.05.01 has an unusual shape with a northeastern shell intersected by a much larger bowl-shaped wing in the southwest (Landecker et al. 1982). The shell has a radius of $25 \mathrm{pc}$, for a distance of $5 \mathrm{kpc}$, and the SNR is currently in its isothermal stage (Pineault et al. 1987). The SNR is interacting with an HI cloud and is inside an expanding HI shell, which yields a more reliable distance of $4.5 \pm 1.5 \mathrm{kpc}$ (Landecker et al. 1989). Burrows \& Guo (1994) present the first X-ray images of VRO.42.05.01: in X-rays it is center-filled. The X-ray flux and temperature yield an improved age: 13000 to $24000 \mathrm{yr}$ for models without and with clouds.

In this paper, OA184 and VRO42.05.01 are mapped at higher sensitivity than previously at $408 \mathrm{MHz}$ and $1420 \mathrm{MHz}$. The resolution is also higher than that for any previous map, except for the $1420 \mathrm{MHz}$ map of Pineault et al. (1987) for VRO42.05.01. The spectral indexes are determined, including an analysis with archival datasets.

\section{Observations and image analysis}

The $408 \mathrm{MHz}$ and $1420 \mathrm{MHz}$ data sets come from the CGPS, which is described in detail by Taylor et al. (2003). The data sets are mainly based on observations from the Synthesis Telescope (ST) of the Dominion Radio Astrophysical Observatory (DRAO). The spatial resolution is better than $1^{\prime} \times 1^{\prime} \operatorname{cosec}(\delta)$ at $1420 \mathrm{MHz}$ and $3.4^{\prime} \times 3.4^{\prime} \operatorname{cosec}(\delta)$ at $408 \mathrm{MHz}$. DRAO ST observations are not sensitive to structures larger than an angular size scale of about $3.3^{\circ}$ at $408 \mathrm{MHz}$ and $56^{\prime}$ at $1420 \mathrm{MHz}$. Thus the CGPS includes data from the $408 \mathrm{MHz}$ all-sky survey of Haslam et al (1982) which has an effective resolution of 51' and the Effelsberg $1.4 \mathrm{GHz}$ Galactic plane survey of Reich et al. $(1990,1997)$ with resolution $9.4^{\prime}$ for large scale emission (the single-dish data are freely available by http://www .mpifr-bonn.mpg.de/survey.html).

We check the OA184 and VRO40.05.01 maps from the $408 \mathrm{MHz}$ all-sky survey, and find that there exists an artifact, 
i.e. low-level striping, that appears as discontinuities across lines of constant Right Ascension. The maximum amplitude of the striping, with a scale of $3^{\circ}$, is less than $5 \%$. In the $408 \mathrm{MHz}$ single-dish map, OA184 is located between the lowest and maximum amplitude of one strip, and is weakly contaminated from 0 in the high RA side to about $5 \%$ in the low RA side by the artifact. VRO40.05.01 is located around the top of one strip, and is contaminated from 3 to $5 \%$ by the artifact. In the $1420 \mathrm{MHz}$ Effelsberg map of the both SNRs, there do not appear to be any significant sidelobe effects.

Estimating the SNRs' flux densities at each frequency is challenging and important for the study of the radio spectrum. We analyze the images and determine flux densities using the DRAO export software package. For the SNRs, integrated flux densities are calculated using the madr routine and errors are found by comparing results for several different choices of background region. For compact sources, the flux densities are calculated using the fluxfit routine, and errors are taken as the formal Gaussian fit errors. The influence of compact sources within the SNRs is very much reduced by employing similar methods to Tian \& Leahy (2005).

\section{Results}

\subsection{Structure at $408 \mathrm{MHz}$ and $1420 \mathrm{MHz}$}

The CGPS images at $408 \mathrm{MHz}$ and $1420 \mathrm{MHz}$ are shown in the upper left and right panels of Fig. 1. OA184 is in the lower half and VRO42.05.01 is in the upper half of each image. The lower left panel shows the $1420 \mathrm{MHz}$ map which has been convolved to the same resolution as the $408 \mathrm{MHz}$ map prior to source removal. For reference we also reproduce the $2695 \mathrm{MHz}$ Effelsberg map (lower right; Fürst et al. 1990) in Fig. 1. The Effelsberg map has an resolution of $4.3^{\prime}$ and a sensitivity of $50 \mathrm{mK} T_{B}$.

The high resolution $408 \mathrm{MHz}$ image for OA184 is the first published at that frequency. Its large elliptical shell-type outline is clearly seen. Due to the high sensitivity and dynamic range of these data, weak diffuse emission of the eastern part of this low brightness SNR is detected. Compact sources distributed across the face of the SNR area are prominent. One large diffuse emission feature just west of OA184 is detected, which has not been reported previously. The $1420 \mathrm{MHz}$ map (expanded view shown in Fig. 2) shows both weak eastern and bright western SNR features much better than previous observations. The features of OA184 at $408 \mathrm{MHz}$ and $1420 \mathrm{MHz}$ are very similar. The weak diffuse region west of OA184 is also seen clearly at $1420 \mathrm{MHz}$, with more detail at $1420 \mathrm{MHz}$ than at $408 \mathrm{MHz}$. This confirms that the new ridge feature is real.

VRO42.05.01 is significantly brighter than OA184. Figure 1 shows the fainter features at 408 and $1420 \mathrm{MHz}$. Figure 2 (right) gives the $1420 \mathrm{MHz}$ higher resolution image with larger range of brightness temperature so that more detail can be seen in the bright regions. The $1420 \mathrm{MHz}$ image best reveals its unusual shape: a northeastern hemispherical "shell" joined to a much larger southwestern bowl-shaped "wing". The brightest parts of the SNR are at the edge of the shell and at the edge of the wing. There is a prominent bridge, not previously noted, along the bottom edge of the shell, seen clearly in Fig. 1 just below the faintest part of the shell interior. The $1420 \mathrm{MHz}$ map in Fig. 2 reveals more diffuse emission than previously seen in both the southeastern and northwestern ends of the wing due to the higher dynamic range of the current data. At $1420 \mathrm{MHz}$ detailed filamentary structure is seen over much of the shell and in the bottom part of the wing region. The $1420 \mathrm{MHz}$ map clearly shows that the boundary between the wing and the shell is aligned parallel to the galactic plane, as described first by Landecker et al. (1982). The radio structures of VRO42.05.01 are the same at $408 \mathrm{MHz}$ and $1420 \mathrm{MHz}$ up to the limit of the resolution of the $408 \mathrm{MHz}$ map.

\section{2. $T-T$ plot spectral indices}

Bright compact sources effect both integrated flux density for the SNRs and spectral indices, we correct for the effects of compact sources below. Each compact source is fit by 1 to 3 Gaussian components plus twisted plane background (details can be found in the DRAO export package manual). Each Gaussian has 6 parameters: $x$ and $y$ positions, normalization, major and minor axes, and orientation of major axis, resulting in up to 18 free parameters fit to each compact source. Table 1 lists properties of the 20 brightest compact sources which are detected within OA184 and VRO42.05.01 at both frequencies.

First we discuss spectral indices between $408 \mathrm{MHz}$ and $1420 \mathrm{MHz}$ based on the $T-T$ plot method. The principle of the $T-T$ plot method is that spectral indices $\left(T_{v}=T_{\circ} v^{-\beta}\right)$ are calculated from a fit of a linear relation to the $T_{1}-T_{2}$ values of all pixels within a given map region. $T_{1}$ is the brightness temperature of a map pixel at one frequency and $T_{2}$ is for the second frequency. The brightness temperature spectral index $\beta$ is derived from the slope of the curve. The flux density spectral index $\alpha\left(\mathrm{S}_{v} \propto v^{-\alpha}\right)$ is related to $\beta$ by $\beta=\alpha+2$. Spectral index refers to flux density spectral index $\alpha$ in this paper unless specifically noted otherwise. The $T-T$ plot method has been widely used for spectral index calculation (e.g., Zhang et al. 1997; Leahy \& Roger 1998).

For the $T-T$ plot analysis, first a single region for the whole of each SNR is used, as shown in Fig. 1. These two regions yield the $T-T$ plots shown in Fig. 3: for OA184 in the top row and for VRO42.05.01 in the bottom row. Three cases are considered: using all pixels including compact sources; using all pixels after subtracting Gaussian fits to the compact sources listed in Table 1 from the images; and excluding compact sources. For OA184 the compact sources are bright compared to the SNR emission. Since the compact sources have a steeper spectrum than the SNR, they are seen in the $T-T$ plot (Fig. 3 upper left panel) as the steeper lines of points extending to higher $T_{B}$. Subtracting compact sources from the image before making the $T-T$ plot removes the lines of points associated with the compact sources (Fig. 3 upper-middle panel). However the Gaussian subtraction is imperfect, leaving artifacts in the $T-T$ plot. There are two reasons why the subtraction is imperfect. First, the maps are made using CLEAN, and bright compact (but not necessarily point) sources can have up to $\approx 100$ CLEAN components, so that with a few (1-3) 

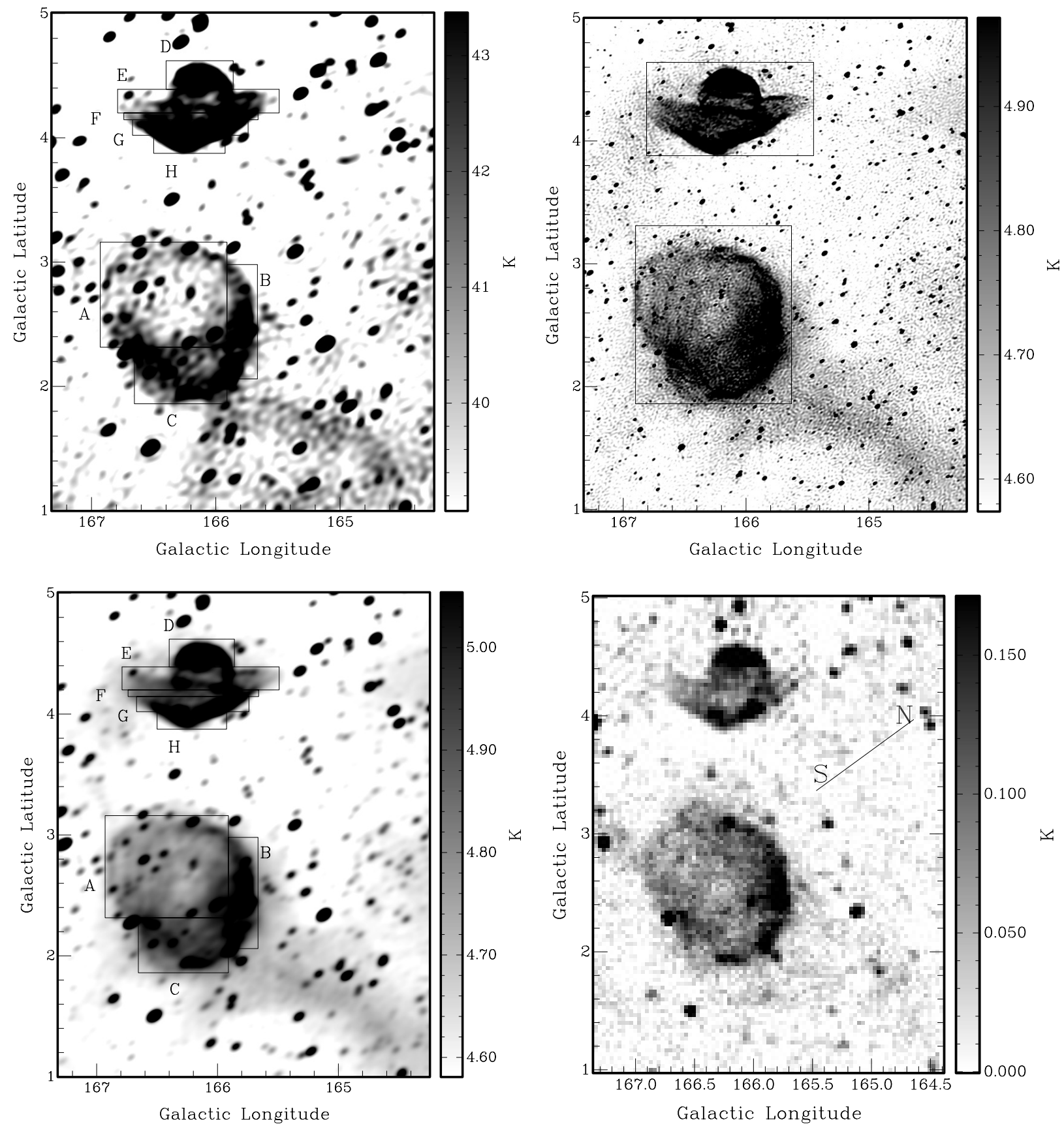

Fig. 1. In each image OA184 is in the lower half and VRO42.05.01 is in the upper half. The first row of images shows the CGPS maps at $408 \mathrm{MHz}$ (left) and $1420 \mathrm{MHz}$ (right). The second row shows the $1420 \mathrm{MHz}$ map convolved to the same resolution as the $408 \mathrm{MHz}$ map (left) and the $2695 \mathrm{MHz}$ Effelsberg map (right). The two boxes used for whole SNR $T-T$ plots are shown in the upper right image. The 8 boxes, labeled with letters and used for SNR sub-areas $T-T$ plots, are shown in the upper and lower left images. The direction of North (N) and South (S) is marked on the lower right image.

Gaussians does not perfectly reproduce the source. Second, the Gaussian fitting routine does not work perfectly in the presence of structured diffuse emission. The next step is to completely remove regions of pixels including the compact sources from the analysis. Each region is taken to be a few beamwidths across, so that any contribution from the compact source is below $1 \%$ of the diffuse SNR emission. Thus any artifacts associated with the compact source are also removed. The last step produces the safest results (upper rightmost plot of Fig. 3). The effects are not as visible for VRO42.05.01 (lower plots of
Fig. 3) since the compact source flux density is much weaker relative to the SNR emission compared to OA184.

Next, the SNRs are subdivided into smaller areas, labeled A to $\mathrm{H}$ in the left panels of Fig. 1, to search for spatial variations in spectral index. Table 2 lists the results for three cases of analysis: including compact sources, subtracting compact sources, and removing compact sources. There is not a large difference in results between the compact sources subtracted and compact sources removed methods. The largest difference is for area B: here there is a $0.7 \sigma$ difference between the two methods. Visual 

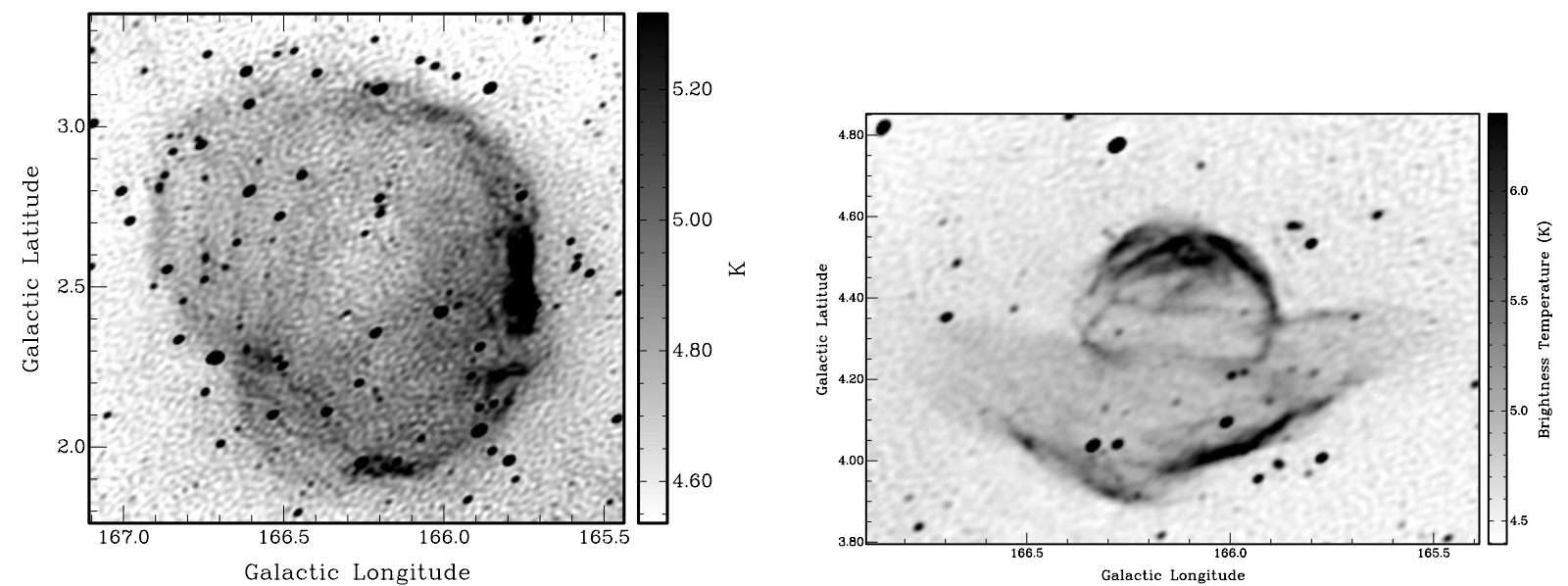

Fig. 2. Expanded $1420 \mathrm{MHz}$ images of OA184 (the left) and VRO42.05.01 (the right) with larger greyscale range.

Table 1. List of brightest compact sources and their Flux Densities $(F D)$ inside OA184 and VRO42.05.01.

\begin{tabular}{cccccc}
\hline \hline Source num. & $\begin{array}{c}\text { GLONG } \\
\text { deg }\end{array}$ & $\begin{array}{c}\text { GLAT } \\
\text { deg }\end{array}$ & $\begin{array}{c}F D \text { at } 408 \mathrm{MHz} \\
\mathrm{mJy}\end{array}$ & $\begin{array}{c}F D \text { at } 1420 \mathrm{MHz} \\
\mathrm{mJy}\end{array}$ & $\begin{array}{c}\text { Sp. index } \\
\alpha\end{array}$ \\
\hline For OA184 & & & & & \\
\hline 1 & 165.887 & 2.055 & $664 \pm 20$ & $229 \pm 9$ & $0.86(0.81$ to 0.90$)$ \\
2 & 165.735 & 2.450 & $309 \pm 33$ & $273 \pm 22$ & $0.10(-0.05$ to 0.25$)$ \\
3 & 166.198 & 3.120 & $196 \pm 8$ & $93 \pm 4$ & $0.60(0.56$ to 0.65$)$ \\
4 & 166.006 & 2.425 & $182 \pm 6$ & $88 \pm 3$ & $0.58(0.54$ to 0.62$)$ \\
5 & 166.605 & 2.803 & $150 \pm 10$ & $60 \pm 3$ & $0.74(0.67$ to 0.81$)$ \\
6 & 166.615 & 3.175 & $121 \pm 7$ & $53 \pm 3$ & $0.66(0.60$ to 0.73$)$ \\
7 & 166.604 & 3.077 & $96 \pm 7$ & $24 \pm 3$ & $1.10(0.99$ to 1.23$)$ \\
8 & 166.211 & 2.359 & $90 \pm 6$ & $36 \pm 2$ & $1.74(1.68$ to 0.81$)$ \\
9 & 166.195 & 2.768 & $59 \pm 4$ & $31 \pm 1$ & $0.67(0.61$ to 0.75$)$ \\
10 & 166.440 & 2.850 & $50 \pm 4$ & $28 \pm 1$ & $0.46(0.40$ to 0.52$)$ \\
11 & 165.990 & 3.036 & $47 \pm 14$ & $14 \pm 5$ & $1.00(0.68$ to 1.53$)$ \\
12 & 166.391 & 3.169 & $45 \pm 5$ & $17 \pm 2$ & $0.78(0.66$ to 0.92$)$ \\
13 & 166.535 & 2.102 & $45 \pm 3$ & $31 \pm 1$ & $0.30(0.25$ to 0.36$)$ \\
14 & 166.821 & 2.345 & $40 \pm 5$ & $26 \pm 1$ & $0.35(0.25$ to 0.47$)$ \\
15 & 166.878 & 2.826 & $39 \pm 10$ & $20 \pm 4$ & $0.54(0.32$ to 0.85$)$ \\
16 & 165.895 & 2.316 & $26 \pm 7$ & $14 \pm 4$ & $0.49(0.24$ to 0.84$)$ \\
17 & 165.926 & 1.839 & $20 \pm 2$ & $10 \pm 1$ & $0.60(0.50$ to 0.71$)$ \\
\hline For VRO42.05.01 & & & & & \\
\hline 1 & 166.327 & 4.024 & $151 \pm 17$ & $70 \pm 10$ & $0.62(0.41$ to 0.83$)$ \\
2 & 166.327 & 4.040 & $73 \pm 16$ & $35 \pm 10$ & $0.59(0.34$ to 0.96$)$ \\
3 & 166.696 & 4.351 & $53 \pm 4$ & $26 \pm 1$ & $0.57(0.50$ to 0.65$)$ \\
\hline
\end{tabular}

inspection of the $T-T$ plots shows that the third method produces the most reliable results, as it did for the entire SNR. The compact sources' influence on the spectral index calculation is obvious in the $T-T$ plots. From now on we discuss spectral indices derived with compact sources removed, unless specified otherwise.

\subsection{Integrated flux densities and spectral indices}

We have derived integrated flux densities for OA184 and VRO42.05.01 from the $408 \mathrm{MHz}$ and $1420 \mathrm{MHz}$ maps. Values given have diffuse background subtracted. The resulting $408 \mathrm{MHz}$ to $1420 \mathrm{MHz}$ spectral indices, using flux densities without compact sources, are $0.25 \pm 0.03$ for OA184 and $0.36 \pm 0.06$ for VRO42.05.01. Table 3 lists the flux densities and spectral indices for both SNRs and flux densities for the compact sources within each SNR. Compact sources contribute about $21 \%$ at $408 \mathrm{MHz}$ and $12 \%$ at $1420 \mathrm{MHz}$ to OA184's flux densities, and have a significant effect on the spectral index of OA184. The effects are significantly smaller for VRO42.05.01. It is noted that the whole SNR spectral indices derived from integrated flux densities is consistent with the whole SNR spectral indices derived by the $T-T$ plot method: $0.23 \pm 0.06$ for OA184 and $0.36 \pm 0.03$ for VRO42.05.01.

Published integrated flux densities and errors for the two SNRs at other frequencies are given in Tables 4 and 5 and shown in Fig. 4. We have calculated total compact source flux densities for these other frequencies, using the $408-1420 \mathrm{MHz}$ spectral index upper and lower limits and flux densities from Table 1. The resulting upper and lower limits to the compact 

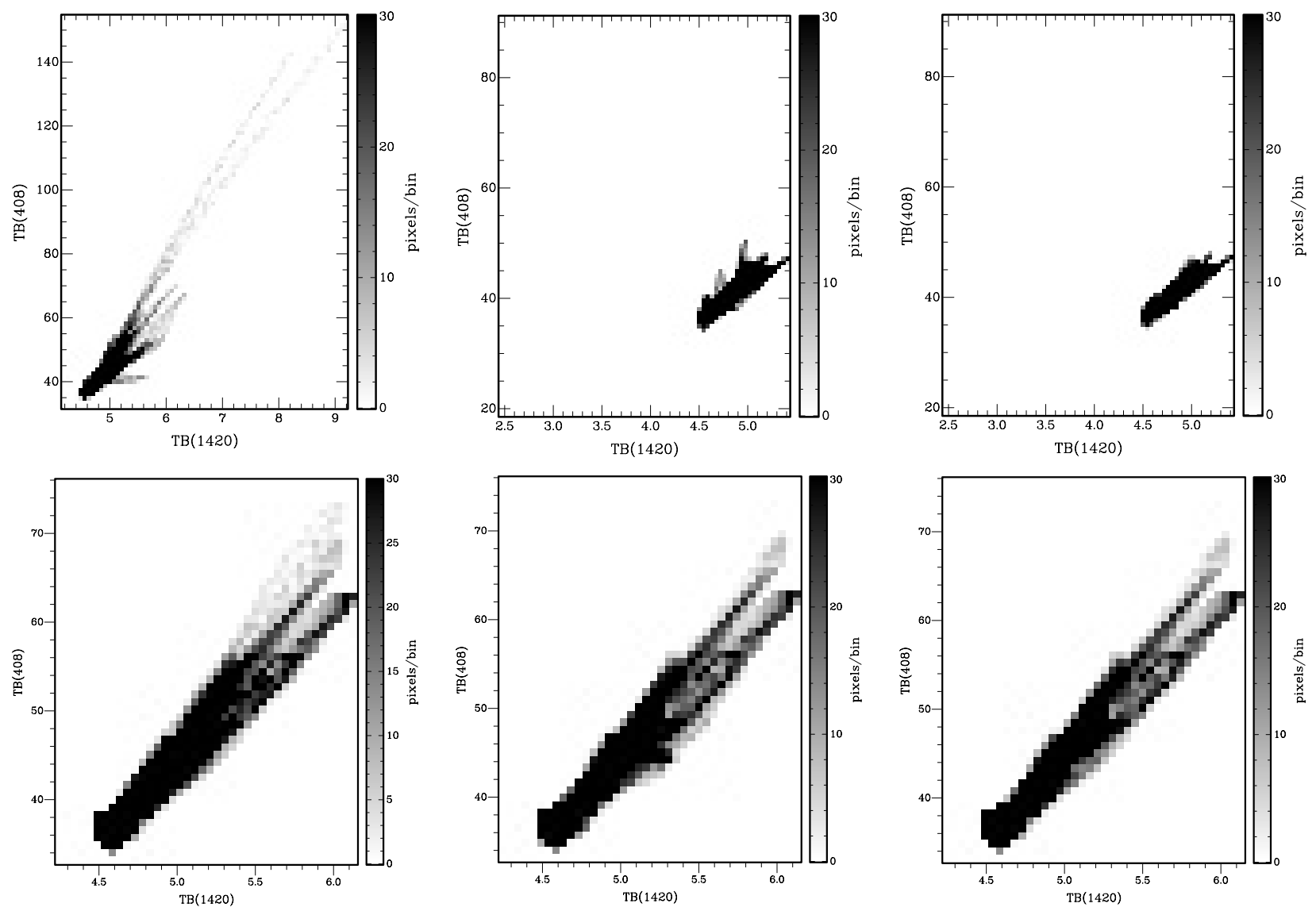

Fig. 3. Whole SNR 408-1420 MHz T - T plots for OA184 (first row) and VRO42.05.01 (second row). From left to right for OA184: plot for map including compact sources $(\alpha=0.28 \pm 0.10)$; plot for map with Gaussian fits to compact sources subtracted $(\alpha=0.21 \pm 0.12)$; plot for compact sources removed from analysis $(\alpha=0.23 \pm 0.06)$. The respective values of $\alpha$ for VRO42.05.01 are $0.38 \pm 0.03,0.36 \pm 0.03$ and $0.36 \pm 0.03$.

Table 2. 408-1420 MHz $T-T$ Plot spectral indices with and without Compact Sources (CS).

\begin{tabular}{cccc}
\hline \hline Sp. index & $\alpha$ & $\alpha$ & $\alpha$ \\
\hline Area & including CS & CS subtracted & CS removed \\
\hline A & $0.43 \pm 0.16$ & $0.21 \pm 0.26$ & $0.22 \pm 0.29$ \\
B & $0.19 \pm 0.04$ & $0.19 \pm 0.10$ & $0.11 \pm 0.05$ \\
C & $0.45 \pm 0.09$ & $0.31 \pm 0.28$ & $0.29 \pm 0.16$ \\
\hline All OA184 & $0.28 \pm 0.10$ & $0.21 \pm 0.12$ & $0.23 \pm 0.06$ \\
\hline D & $0.31 \pm 0.01$ & $0.31 \pm 0.01$ & $0.31 \pm 0.01$ \\
E & $0.35 \pm 0.06$ & $0.39 \pm 0.11$ & $0.39 \pm 0.08$ \\
F & $0.53 \pm 0.04$ & $0.53 \pm 0.04$ & $0.53 \pm 0.04$ \\
G & $0.49 \pm 0.02$ & $0.44 \pm 0.01$ & $0.45 \pm 0.01$ \\
H & $0.48 \pm 0.01$ & $0.47 \pm 0.01$ & $0.47 \pm 0.01$ \\
\hline All VRO42.05.01 & $0.38 \pm 0.03$ & $0.36 \pm 0.03$ & $0.36 \pm 0.03$ \\
\hline
\end{tabular}

source flux densities within OA184 are shown in Fig. 4 (left). For VRO42.04.01 the compact source flux densities are much smaller, so are not plotted in order to show the SNR flux densities more clearly. For OA184, the compact sources' flux density relative to OA184 plus compact sources (plus ridge when applicable, see below) is the highest at $38 \mathrm{MHz}$ at $13-22 \%$. It smoothly decreases to $10-13 \%$ at $2695 \mathrm{MHz}$. For VRO42.04.01, it ranges from $5-9 \%$ at $38 \mathrm{MHz}$ to $1.5-2 \%$ at $2695 \mathrm{MHz}$. The published flux densities need to have the compact sources' flux density contribution subtracted in order to study the spectrum of the SNRs.

For OA184, in addition to the effect of compact sources, there exists the ridge emission region just west of the SNR, as seen clearly in Fig. 1 at $408 \mathrm{MHz}$ and $1420 \mathrm{MHz}$. The ridge is also detected in the Effelsberg $2695 \mathrm{MHz}$ map. The low resolution published maps include this ridge emission as part of OA184. For example, the $408 \mathrm{MHz}$ all-sky survey map of Haslam et al. (1982) (with $51 \times 51 \operatorname{arcmin}^{2}$ beam) has a single bright area including both OA184 and ridge. The $38 \mathrm{MHz}$, $102 \mathrm{MHz}$ and, to a lesser extent, $610 \mathrm{MHz}$ flux densities need to be corrected for the ridge. The reality of this correction is supported by 4.8 Jy flux density difference between our high resolution $408 \mathrm{MHz}$ flux density value and Haslam's low resolution $408 \mathrm{MHz}$ flux density value (both given in Table 4): $2.2 \mathrm{Jy}$ of the difference is due to compact sources the other $2.6 \mathrm{Jy}$ is due to the ridge (see next paragraph).

We measure the brightness temperatures of the ridge: $\simeq 2.6 \mathrm{~K}$ at $408 \mathrm{MHz}, \simeq 0.12 \mathrm{~K}$ at $1420 \mathrm{MHz}$, and $\simeq 0.02 \mathrm{~K}$ in the Effelsberg $2695 \mathrm{MHz}$ map. The brightness temperatures give the ridge spectral index $\alpha \simeq 0.5(408-1420 \mathrm{MHz})$, $\alpha \simeq 0.6(1420-2695 \mathrm{MHz})$ and $\alpha \simeq 0.8(408-2695 \mathrm{MHz})$. These are consistent with eachother within the uncertainties of measurement of the ridge brightness temperatures. The ridge emission region has steeper radio spectrum than the SNR so it 
Table 3. Integrated flux densities and spectral indices of OA184, VRO42.05.01, and compact sources within OA184 and VRO42.05.01.

\begin{tabular}{ccccccc}
\hline \hline Freq. & OA184 & CS of OA184 & OA184+CS & VRO42 & CS of VRO42 & VRO42 +CS \\
MHz & $\mathrm{Jy}$ & $\mathrm{Jy}$ & $\mathrm{Jy}$ & $\mathrm{Jy}$ & $\mathrm{Jy}$ & $\mathrm{Jy}$ \\
\hline 408 & $10.7 \pm 1.0$ & $2.2 \pm 0.2$ & $12.9 \pm 1.2$ & $8.1 \pm 1.0$ & $0.28 \pm 0.04$ & $8.4 \pm 1.0$ \\
1420 & $7.8 \pm 0.3$ & $1.0 \pm 0.1$ & $8.8 \pm 0.4$ & $5.2 \pm 0.2$ & $0.13 \pm 0.02$ & $5.3 \pm 0.2$ \\
\hline$\alpha$ & $0.25 \pm 0.03$ & $0.63 \pm 0.12$ & $0.31 \pm 0.04$ & $0.36 \pm 0.06$ & $0.62 \pm 0.12$ & $0.37 \pm 0.05$ \\
\hline
\end{tabular}

Table 4. Integrated flux densities $(F D)$ of OA184.

\begin{tabular}{|c|c|c|c|}
\hline $\begin{array}{l}\text { Freq. } \\
\text { MHz }\end{array}$ & $\begin{array}{c}\text { Beamwidth } \\
\text { arcmin }\end{array}$ & $\begin{array}{r}F D \\
\text { Jy }\end{array}$ & References \\
\hline 38.0 & $45 \times 45$ & $70.0^{*} \pm 20.0$ & Haslam \& Salt \\
\hline 102.0 & $48 \times$ & $37.0 \pm 6.0$ & Kovalenko et al. (1994) \\
\hline 408.0 & $3.4 \times$ & $10.7 \pm 1.0$ & this \\
\hline 408.0 & $45 \times 45$ & $15.5 \pm 4.0$ & Haslam \& Salter (1971) \\
\hline 610.0 & $15 \times$ & $13.5 \pm 1.5$ & Dickel \& Yang (1965) \\
\hline 1420.0 & $1>$ & $9.0 \pm 0.5$ & Routledge et al. (1986) \\
\hline 1420.0 & $1 \times$ & $7.8 \pm 0.3$ & this paper \\
\hline 2695 & $3.4 \times 3.4$ & $6.9 \pm 0.7$ & see text \\
\hline 2700.0 & $5 \times 5$ & $6.0 \pm 2.2$ & Willis (1973) \\
\hline
\end{tabular}

*Corrected using scale correction of Roger et al. (1973).

Table 5. Integrated flux densities $(F D)$ of VRO42.05.01.

\begin{tabular}{cccc}
\hline \hline $\begin{array}{c}\text { Freq. } \\
\text { MHz }\end{array}$ & $\begin{array}{c}\text { Beamwidth } \\
\text { arcmin }\end{array}$ & $\begin{array}{c}F D \\
\mathrm{Jy}\end{array}$ & References \\
\hline 38.0 & $45 \times 45$ & $22.4^{*}$ & Williams et al. (1966) \\
83.0 & $59 \times 31$ & $20.0 \pm 7.0$ & Kovalenko et al. (1994) \\
408.0 & $45 \times 45$ & $9.0 \pm 1.6$ & Haslam \& Salter (1971) \\
408.0 & $3.4 \times 5.1$ & $8.1 \pm 1.0$ & this paper \\
610.0 & $15 \times 15$ & $7.5 \pm 1.0$ & Dickel et al. (1965) \\
1400.0 & $10 \times 10$ & 6.8 & Felli \& Churchwell (1972) \\
1420.0 & $1 \times 1.4$ & $6.0 \pm 0.8$ & Landecker et al. (1982) \\
1420.0 & $1 \times 1.5$ & $5.2 \pm 0.2$ & this paper \\
2965 & $3.4 \times 3.4$ & $4.6 \pm 0.2$ & see text \\
2700.0 & $5 \times 5$ & $5.2 * * \pm 1.0$ & Willis (1973) \\
\hline
\end{tabular}

*Corrected using scale correction of Roger et al. (1973).

**Probably high due to inclusion of compact sources to the northeast.

contributes more flux density to OA184 at low frequencies than at high frequencies. The ridge flux densities at different frequencies are calculated using the flux density at $408 \mathrm{MHz}$ and spectral index of $\alpha=0.5-0.8$. The ridge region flux density is $9-17 \mathrm{Jy}$ at $38 \mathrm{MHz}, 5-8 \mathrm{Jy}$ at $102 \mathrm{MHz}$ and $1.9-2.1 \mathrm{Jy}$ at $610 \mathrm{MHz}$.

Thus we have recalculated the flux density values of OA184: at $38 \mathrm{MHz}, 102 \mathrm{MHz}$ and $610 \mathrm{MHz}$ we subtract the ridge flux density and the compact source flux density; at $2695 \mathrm{MHz}$ we only subtract the compact source flux density. For 408 and $1420 \mathrm{MHz}$ the values in Table 4 already have compact source flux density removed and we have already subtracted the ridge flux density by including it in the background calculation. For VRO42.05.01, only the compact sources' flux density corrections are needed at 38, 83, 610 and $2700 \mathrm{MHz}$. We note that Landecker et al. (1982) believe that the flux density at $2700 \mathrm{MHz}$ from Willis (1973) is overestimated. Thus we obtain a new flux density value from the Effelsberg $2695 \mathrm{MHz}$ image: $4.6 \pm 0.2 \mathrm{Jy}$ and use this instead. We fit the resulting flux density values with a power-law to obtain spectral index. Figure 3 shows the corrected flux densities and the best-fit power-law. OA184 has a best fit spectral index of $\alpha=0.32 \pm 0.06, \chi^{2}=2.3$, when we use a ridge spectral index of 0.8 or $\alpha=0.34 \pm 0.06, \chi^{2}=4.2$, when we use a ridge spectral index of 0.5. If we omit the most uncertain flux densities of OA184 at the two lowest frequencies, the best-fit spectral index is $0.28 \pm 0.06, \chi^{2}=0.4\left(\alpha_{\text {ridge }}=0.5\right.$ or 0.8$)$. VRO42.05.01 has a best-fit spectral index of $0.32 \pm 0.05, \chi^{2}=2.3$.

\section{Discussion}

\subsection{OA184}

Low spatial resolution observations of OA184 included the flux density from the ridge west of OA184 as part of OA184. The ridge spectral index of 0.5 to 0.8 is derived from the flux densities in our 408 and $1420 \mathrm{MHz}$ maps and in the 2695 Effelsberg map. The ridge contributes $\simeq 1 / 3$ of the flux density from OA184 in the low frequency maps. OA184 also has a significant contribution from compact sources within its boundary. At $408 \mathrm{MHz}$ it is $21 \%$ and at $1420 \mathrm{MHz}$ it is $12 \%$ (Table 3). At lower frequencies the contribution is higher: e.g. at $38 \mathrm{MHz}$ it is $\simeq 17 \%$ of the total or $\simeq 30 \%$ of the OA184 flux density. Thus both compact source and ridge emission corrections are necessary to study the radio spectrum of OA184. The resulting multi-frequency spectral index is $0.32 \pm 0.06$. This is consistent with the $408 \mathrm{MHz}-1420 \mathrm{MHz}$ spectral index derived using an entire SNR $T-T$ plot method $(\alpha=0.23 \pm 0.06)$ or using integrated flux densities $(\alpha=0.25 \pm 0.03)$.

OA184 is split into smaller regions: A (east), B (northwest rim), and C (southwest rim, see Fig. 1) to look for spectral index variations. The northwest rim (B in Table 2 ) is found to have a marginally smaller spectral index (by $1.5 \sigma$ ) than whole SNR spectral index. Since OA184 is an old remnant (Leahy \& Marshall 1988), the shock has slowed down to a mach number of only a few. Where the shock collides with a cooler denser ISM, the mach number will increase resulting in a flatter electron spectrum and spectral index, as well as a brightening. Both are observed at the northwest rim. However, as discussed in Leahy \& Roger (1998), the effect of mach number on spectral index is only effective for $\alpha>0.5$ for linear shock acceleration theory. Non-linear effects in shock acceleration are poorly understood but can reduce spectral index. The variable spectral index with $\alpha<0.5$ could be due to non-linear effects, absorption or an admixture of thermal radiation which is 

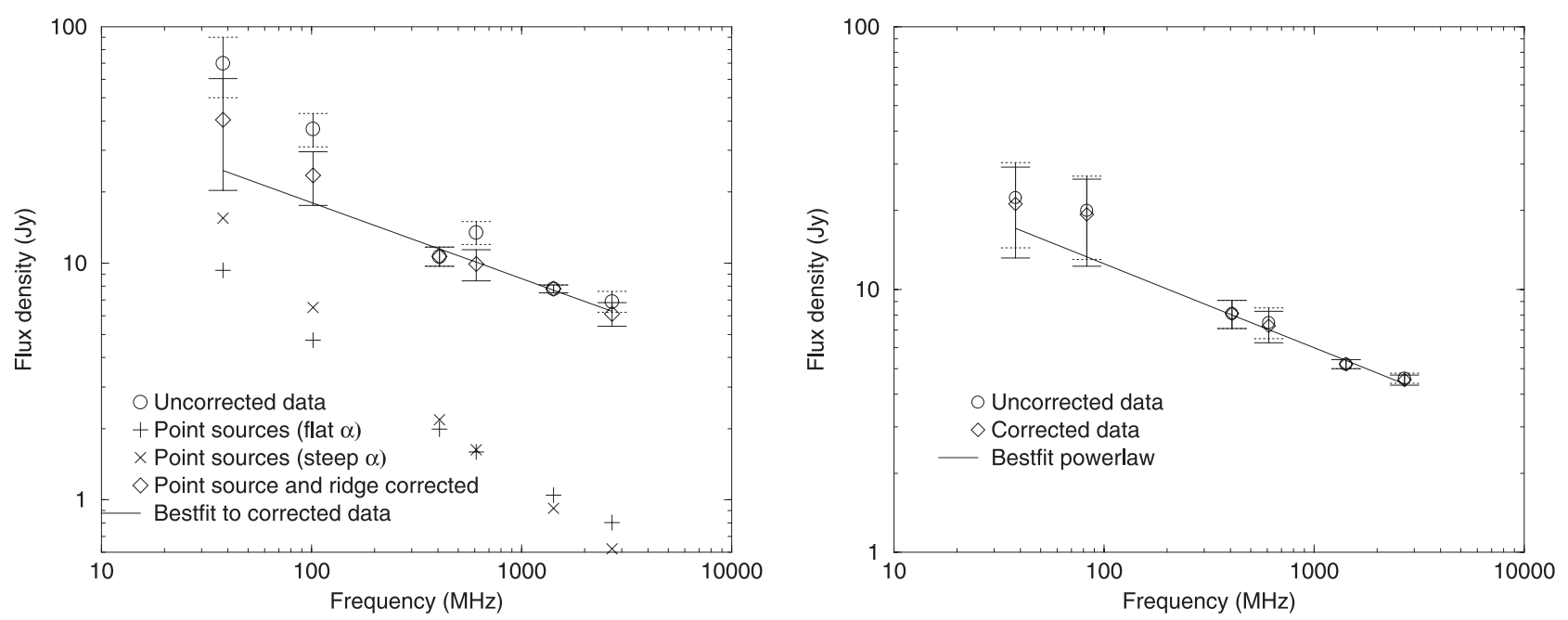

Fig. 4. Radio spectrum of OA184 (left) and VRO42.05.01 (right). OA184 has a best-fit spectral index of $0.32, \chi^{2}=2.3$ for data corrected for the ridge emission $\alpha_{\text {ridge }}=0.8$ and compact sources. VRO42.05.01 has best fit spectral index of $0.32, \chi^{2}=2.3$ for data corrected for compact sources.

flattening the spectrum. The pre-shock density of OA184 was derived by Leahy \& Marshall (1988) as $0.2 \mathrm{~cm}^{-3}$. This low value and the lack of a low-frequency turnover in the radio spectrum rule out absorption effects, which have a strong effect on the low-frequency radio spectrum (Leahy \& Roger 1998).

\section{2. $V R 042.05 .01$}

Compact sources only contribute a few percent to the flux density of VRO42.05.01 at all frequencies. The mean spectral index of VRO42.05.01 is 0.36 (Table 3). Yet there are spectral index variations within this SNR detected at high significance. Evidence for this is already seen in Fig. 3, lower panels, where several lines of points of different slopes are seen. For the five subregions (D through $\mathrm{H}$, see Fig. 1), the $T-T$ plots have single lines of points with well determined slopes (except region E), as given in Table 2. The northeast shell, region $\mathrm{D}$, has the lowest value of $\alpha, 0.31$, and the southwest wing, regions $\mathrm{F}, \mathrm{G}$ and $\mathrm{H}$, have high values, $0.45-0.53$. The boundary region between the shell and the wing, region $\mathrm{E}$, has a $T-T$ plot consistent with a mixture of $\alpha=0.31$ and 0.47 values.

This spatial variation of spectral index is consistent with the flux density vs. frequency plot. The flux density values (Fig. 4, right) are adequately fit by a single power law with $\alpha=0.32$, yet it is fit slightly better by a sum of two power laws with spectral indices taken from the subregions: $\alpha=0.31$ (from D) and $\alpha=0.47$ (from $\mathrm{F}, \mathrm{G}$ and $\mathrm{H}$ ). The possible causes for variable $\alpha<0.5$ are the same as listed for OA184. For VRO42.05.01, the pre-explosion density is best determined by X-ray observations and is low -0.01 to $0.002 \mathrm{~cm}^{-3}$ (Burrows \& Guo 1994). This and lack of low-frequency turnover in the radio spectrum rule out absorption mechanisms. Also, the fact that the shell and wing regions have different mach numbers and different values of $\alpha$ is very suggestive that the spectral index changes are due to difference in mach number. To get the spectral indices below 0.5 requires also non-linear shock effects. Further theoretical studies on shock acceleration are worthwhile.

\section{Conclusion}

We present new images of the SNRs OA184 and VRO42.05.01 at $408 \mathrm{MHz}$ and $1420 \mathrm{MHz}$ from the CGPS data sets The SNRs' flux densities at both $408 \mathrm{MHz}$ and $1420 \mathrm{MHz}$ are found and corrected for flux density from compact sources within the SNRs. The resulting integrated flux density based spectral index is $0.36 \pm 0.06$ for VRO42.05.01. This agrees with the $T-T$ plot spectral index of $0.36 \pm 0.03$ and with the $38 \mathrm{MHz}$ to $2695 \mathrm{MHz}$ spectral index of $0.32 \pm 0.05$ from published flux densities corrected for compact sources. We find that the shell region of VRO42.05.01 has $\alpha=0.3$ and that the wing region has $\alpha=0.47$.

For OA184, the integrated flux density based spectral index, $0.25 \pm 0.03$, agrees with the $T-T$ plot spectral index, $0.23 \pm 0.06$. However, these are smaller than previously published values. A new ridge emission region is detected just west of OA184, which was included in lower resolution maps as part of OA184. Subtracting the ridge and compact source contributions yields a spectral index of $0.32 \pm 0.06$ (for flux densities from $38 \mathrm{MHz}$ to $2695 \mathrm{MHz}$ ) or $0.28 \pm 0.06$ (for flux densities from $408 \mathrm{MHz}$ to $2695 \mathrm{MHz}$ ). These are now consistent with our $408-1420 \mathrm{MHz}$ spectral indices. Spatial variations of spectral index are found in OA184: $\sim .1$ for the bright northwest edge and $\sim 0.3$ for the rest of the SNR, although the large uncertainties make an accurate determination of this difference difficult.

Acknowledgements. We acknowledge support from the Natural Sciences and Engineering Research Council of Canada. W.W. Tian thanks the National Natural Science Foundation of China for support. The DRAO is operated as a national facility by the National Research Council of Canada. The Canadian Galactic Plane Survey is a Canadian project with international partners.

\section{References}

Burrows, D. N., \& Guo, Z. Y. 1994, ApJ, 421, L19

Dickel, J. R., McGuire, J. P., \& Yang, K. S. 1965, ApJ, 142, 798 
Dickel, J. R., \& Yang, K. S. 1965, ApJ, 142, 1642

Fürst, E., Reich, W., Reich, P., \& Reif, K. 1990, A\&AS, 85, 691

Haslam, C. G. T., Salter, C. J., Stoffel, H., \& Wilson, W. W. 1982, A\&AS, 47, 1

Haslam, C. G. T., \& Salter, C. J. 1971, MNRAS, 151, 385

Huang, Y., \& Thaddeus, P. 1986, ApJ, 309, 804

Kovalenko, A. V., Pynzar, A. V., \& Udal'tsov, V. A. 1994, AR, 38, 95

Landecker, T. L., Pineault, S., Routledge, D., \& Vaneldik, J. F. 1989, MNRAS, 237, 277

Landecker, T. L., Pineault, S., Routledge, D., \& Vaneldik, J. F. 1982, ApJ, 261, L41

Leahy, D. A., \& Marshall, C. R. 1988, MNRAS, 235, 805

Leahy, D. A., \& Roger, R. S. 1998, ApJ, 505, 784
Felli, M., \& Churchwell, E., 1972, A\&AS, 5, 369

Pineault, S., Landecker, T. L., \& Routledge, D. 1987, ApJ, 315, 580

Reich, W., Reich, P., \& Fürst, E. 1990, A\&AS, 83, 539

Reich, W., Reich, P., \& Fürst, E. 1997, A\&AS, 126, 413

Roger, R. S., Costain, C. H., \& Bridle, A. H. 1973, AJ, 78, 1030

Routledge, D., Landecker, T. L., \& Vaneldik, J. F. 1986, MNRAS, 221, 809

Taylor, A. R., Gibson, S. J., \& Peracaula, M. 2003, AJ, 125, 3145

Tian, W. W., \& Leahy, D. A. 2005, A\&A, 436, 187

Williams, P., Kenderkine, S., \& Baldwin, J. 1966, MmRAS, 70, 53

Willis, A. G. 1973, A\&A, 26, 237

Zhang, X. Z., Zheng, Y., \& Landecker, T. L., et al. 1997, A\&A, 324, 641 